\title{
Small cell lung cancer in never-smokers
}

\author{
María Torres-Durán ${ }^{1}$, Alberto Ruano-Ravina ${ }^{2,3,4}$, Karl T. Kelsey ${ }^{4}$, \\ Isaura Parente-Lamelas ${ }^{5}$, Mariano Provencio ${ }^{6}$, Virginia Leiro-Fernández ${ }^{1}$, \\ José Abal-Arca ${ }^{5}$, Carmen Montero-Martínez ${ }^{7}$, Iria Vidal-Garcia ${ }^{7}$, Carolina Pena ${ }^{8}$, \\ Olalla Castro-Añón ${ }^{9}$, Antonio Golpe-Gómez ${ }^{10}$, Cristina Martínez ${ }^{11}$, \\ Rosirys Guzmán-Taveras ${ }^{11}$, María José Mejuto-Martí ${ }^{12}$, \\ Alberto Fernández-Villar ${ }^{1}$ and Juan Miguel Barros-Dios ${ }^{2,3,13}$
}

\begin{abstract}
Affiliations: ${ }^{1}$ Service of Pneumology, University Hospital Complex of Vigo, Vigo, Spain. ${ }^{2}$ Dept of Preventive Medicine and Public Health, University of Santiago de Compostela, Santiago de Compostela, Spain. ${ }^{3}$ CIBER de Epidemiología y Salud Pública (CIBERESP), Spain. ${ }^{4}$ Dept of Epidemiology, Brown School of Public Health, Brown University, Providence, Rhode Island, USA. ${ }^{5}$ Service of Pneumology, Ourense Hospital Complex, Ourense, Spain. ${ }^{6}$ Service of Oncology, Puerta de Hierro University Hospital, Madrid, Spain. ${ }^{7}$ Service of Pneumology, University Hospital Complex of A Coruna, A Coruna, Spain. ${ }^{8}$ Service of Oncology, Oncologic Center of A Coruna, A Coruna, Spain. 'Service of Pneumology, Hospital Lucus Augusti, Lugo, Spain. ${ }^{10}$ Service of Pneumology, Santiago de Compostela University Clinic Hospital, Santiago de Compostela, Spain. ${ }^{11}$ National Institute of Silicosis, University Hospital of Asturias, Oviedo, Spain. 12Service of Pneumology, Arquitecto Marcide Hospital, Ferrol, Spain. ${ }^{13}$ Service of Preventive Medicine, University Hospital Complex of Santiago de Compostela, Santiago de Compostela, Spain.
\end{abstract}

Correspondence: A. Ruano-Ravina, Dept of Preventive Medicine and Public Health, School of Medicine, C/ San Francisco s/n, University of Santiago de Compostela, 15782 Santiago de Compostela, Spain.

E-mail: alberto.ruanodusc.es

ABSTRACT Our aim was to describe the characteristics of a case-series of never-smoker small cell lung cancer (SCLC) cases.

Cases of SCLC were selected from a prospective, multicenter, hospital-based case-control study performed in Spain. Participants were never-smokers older than 30 years with an anatomo-pathological confirmation of primary lung cancer. We collected clinical and epidemiological variables according to the study's protocol.

We included 19 SCLC cases, 18 females (94.7\%), median age 75 years (interquartile range (IQR) 70-80 years). Median residential radon concentration was $195 \mathrm{~Bq} \cdot \mathrm{m}^{-3}$ (IQR $130-229 \mathrm{~Bq} \cdot \mathrm{m}^{-3}$ ). 10 patients had limited disease and nine had extended disease. Median survival was 242 days (IQR 94-496 days); 1- and 2-year survival were $36.8 \%$ and $17.6 \%$, respectively. Survival was much higher for individuals with limited disease than for those with extended disease (median 336 versus 235 days; 1 -year survival $50 \%$ versus $22.2 \%$ and 2 -year survival $27 \%$ versus $0 \%$, respectively). Performance status at diagnosis was closely related to survival.

SCLC is an infrequent, highly aggressive disease in never-smokers. Survival is poor, even for limited disease. Age at diagnosis in SCLC is higher than that observed for never-smokers with adenocarcinoma. Residential radon exposure is higher than the action levels recommended by the World Health Organization.

@ERSpublications

Small cell lung cancer in never-smokers has a poor survival and is diagnosed at older ages than other lung cancers http://ow.ly/USS2W

Received: Sept 112015 | Accepted after revision: Nov 12 2015 | First published online: Dec 232015

Support statement: This paper has been funded by a competitive research grant of the Xunta de Galicia: 10CSA208057PR "Risk factors of lung cancer in never smokers: a multicenter case-control study in the Northwest of Spain." It has also received partial funding from the grant of the Instituto de Salud Carlos III "Small Cell Lung Cancer, risk factors and genetic susceptibility. A multicentre case-control study in Spain (Small Cell Study) (REF PI15/01211)." Part of this work was performed during a Fulbright grant for Senior Researchers awarded to A. Ruano-Ravina at Brown University (Providence, Rhode Island, USA) (REF PRX14/00365). This grant is awarded by the US Department of State in collaboration with the Spanish Ministry of Education. Funding information for this article has been deposited with FundRef.

Conflict of interest: None declared.

Copyright @ERS 2016 


\section{Introduction}

Lung cancer is an important health problem in many countries. An estimated 1.6 million new cases are diagnosed worldwide every year and it is the leading cause of cancer mortality worldwide with $>1400000$ annual deaths [1]. This disease has different histological types and the most frequent are, in order: adenocarcinoma, squamous cell carcinoma, small cell lung cancer (SCLC) and large cell carcinoma [2], although there are other infrequent histologies. The histologic pattern of lung cancer has changed in recent years, with squamous cell lung cancer being surmounted by adenocarcinoma, probably due to smoking cessation trends and changes in tobacco composition [3]. Due to its particular characteristics, lung neoplasms have been defined as non-SCLCs (NSCLCs), including all histological types apart from SCLC, and SCLCs [4].

SCLC comprises $\sim 15 \%$ of all lung cancer cases and it is tightly linked with tobacco consumption [5]. It is extremely infrequent in never-smokers. In an epidemiologic study performed in the USA, only $2.5 \%$ of all SCLC cases were diagnosed among never-smokers [6]. SCLC originates in neuroendocrine cells and is characterised for being a rapidly growing tumour, which has an initial high rate of response to treatment, although it is resistant to treatment in those cases where metastatic disease is present [5].

Risk factors for SCLC in never-smokers are completely unknown, although it is supposed that environmental tobacco smoke (ETS) exposure can play a role [7], as well as some risk arising from occupational exposures. There is also little knowledge about patterns of age at onset, gender distribution and stage at diagnosis (limited or extended disease) in these patients.

Information is also scarce on the role that residential radon might play in the genesis of SCLC in never-smokers. Some studies have found that residential radon could be more tightly related with SCLC compared with other lung cancer histological types [8]. Nevertheless, a study analysing residential radon and histological types in never-smokers observed that residential radon concentrations in SCLC patients were lower than that observed for other histological types [9].

Here, we aim here to describe the characteristics of a case-series of never-smoking SCLC patients from the Lung Cancer Risk in Never Smokers Study (LCRINS study), a study performed in the northwest of Spain, which is a radon-prone area.

\section{Materials and methods}

\section{Design and setting}

Cases of SCLC were selected from the LCRINS study, which was designed as a multicenter, hospital-based, case-control study. Participants were recruited over 36 months, between January 2011 and December 2014, in nine Spanish hospitals (seven in Galicia, one in Asturias and one in Madrid). The participating hospitals were: Vigo University Hospital, Santiago de Compostela University Hospital, A Coruna University Hospital, Lucus Augusti University Hospital, Ourense Hospital Complex, Pontevedra Hospital Complex, Arquitecto Marcide Hospital, Central University Hospital of Asturias and Puerta de Hierro University Hospital. All consecutive lung cancer cases diagnosed in never-smokers at the participating hospitals were included. All cases had pathological confirmation of primary lung cancer.

All participants were never-smokers. This definition followed that proposed by the World Health Organization (WHO): individuals that had smoked 1) $<100$ cigarettes in their lifetime or b) never as much as one cigarette per day during a consecutive period of 6 months. Participants had to be older than 30 years and without a previous cancer history to be included.

The study protocol was approved by the Galician Committee of Research Ethics (reference 2010/295). Written consent was obtained from all participants.

\section{Data collection}

All participants underwent an interview by trained researchers using a specific questionnaire. They were asked about different issues of their lifestyle, including exposure to ETS, leisure time activities, occupational exposure, and characteristics related to their dwellings and radon exposure. Their electronic medical records were checked to identify their symptoms at the onset of the disease and to determine if the disease was limited or extended at the diagnosis. Performance status was assessed for all patients at diagnosis using the Eastern Cooperative Oncology Group Study Performance Status Scale [10].

Residential radon measurement was performed at the participant's dwelling by installing an alpha track-type radon detector (CR-39; Radosys, Budapest, Hungary). The detector was in place for a minimum of 3 months, mainly in the master bedroom. We gave detailed instructions to participants on how to place radon devices, including a descriptive picture showing where the device should be placed. They also received a prepaid and easy-to-seal envelope to send back the detector after the end of the measurement period. 
We reminded them through a phone call to return the radon detector when the 3-month period ended. Devices were read at the Galician Radon Laboratory, which has participated in intercomparison activities with excellent results [11] and has been certified by the University of Cantabria Radon Laboratory [12]. We used seasonal adjustment when obtaining radon results and these results were sent back to participants.

\section{Statistical analysis}

We performed a descriptive analysis to characterise the clinical characteristics of SCLC cases. We calculated the median residential radon concentration for all cases and also considering if SCLC was limited or extended at diagnosis. We also assessed the survival of these patients using Kaplan-Meier survival analysis and broke down results by extension at diagnosis. The statistical analysis was performed with SPSS Statistics (Windows, version 21.0; IBM, Armonk, NY, USA).

\section{Results}

We recruited 19 SCLC cases from a total of 322 never-smoking lung cancer cases (5.9\%). Of these, 18 were females $(94.7 \%)$. The age range was $53-86$ years with a median of 75 years (interquartile range (IQR) 70-80 years). The characteristics of these patients are described in table 1.

Regarding stage at diagnosis, 10 cases had limited disease (confined to the thorax) and nine had extended disease. Only four participants reported having lived with a smoker during the last 20 years in the same dwelling. Most of the participants were housewives or performed agricultural activities. An additional four participants had worked as cleaners. No study participant reported performing do-it-yourself activities such as model-making, furniture refinishing (including varnishing) or painting (artistic or not).

Median residential radon was $195 \mathrm{~Bq} \cdot \mathrm{m}^{-3}$ (IQR $130-229 \mathrm{~Bq} \cdot \mathrm{m}^{-3}$ ). The range of radon measurements was $25-1430 \mathrm{~Bq} \cdot \mathrm{m}^{-3}$. This radon concentration is higher than the median radon concentration observed in never-smoking controls of the LCRINS study $\left(149 \mathrm{~Bq} \cdot \mathrm{m}^{-3}\right)$. The nonsmoking cases lived for a median time of 43 years in the same dwelling compared with 36 years for controls. Median radon concentration of SCLC cases with limited disease was $190 \mathrm{~Bq} \cdot \mathrm{m}^{-3}$ compared with $195 \mathrm{~Bq} \cdot \mathrm{m}^{-3}$ for those with extended disease.

The most frequent symptoms reported at diagnosis are described in table 2. All patients with constitutional syndrome had an extended disease at diagnosis, whereas patients with limited disease presented symptoms related to local affectation (cough, haemoptysis, thoracic pain or dyspnoea), with cough being the most frequent symptom (present in $58 \%$ of patients at diagnosis). In one patient a "syndrome of inappropriate antidiuretic hormone secretion" was detected at the time of diagnosis of SCLC and another patient presented a pruritus sine materia; both were interpreted as paraneoplasic syndromes. Brain metastasis was detected in one patient at SCLC diagnosis.

Performance status broken by stage disease at diagnosis is described in table $3.70 \%$ of patients with limited disease had a performance status of $0-1$ and $20 \%$ had a performance status of $3-4$. $56 \%$ of patients with extended disease had a performance status of $0-1$ and the remaining patients with extended disease had a performance status of 3-4.

Regarding survival, the median time was 242 days (IQR 94-496 days); 1 - and 2-year survival were 36.8\% and $17.6 \%$, respectively. Median survival was higher for participants diagnosed with limited disease (336 days (IQR 94 days-alive)) compared with those diagnosed with extended disease (235 days (IQR 210283 days)). By stage at diagnosis, 1-year survival was $50 \%$ for limited disease and $22.2 \%$ for extended disease, and 2 -year survival was $27 \%$ for limited disease and $0 \%$ for extended disease. Regarding performance status, median survival was much higher for individuals with a performance status $\leqslant 2$ (336 days (IQR 229 days-alive)) than for those with performance status $\geqslant 3$ (58 days (IQR 32-235 days)).

\section{Discussion}

SCLC in never-smokers is a very infrequent disease that might have particular characteristics. In our case-series, the only risk factor that appeared to be associated with this specific cancer subtype was residential radon, as risk occupations for lung cancer or exposure to ETS were not frequently reported by our participants. The concentration of radon reported by the participants was higher than that observed in the general population recruited in the same area. Of note, SCLC in never-smokers seemed to be diagnosed later in life than other lung cancer histological types, at a median age of 75 years. Survival for never-smoking SCLC cases is extremely low, similar to their smoking counterparts [13].

This case-series studied one of the largest sample sizes reported to date. Adenocarcinoma is the most common histological type in never-smokers. In research published in 2001 by LAGARDE et al. [14] comprising 258 never-smoker lung cancer cases in patients exposed to indoor radon, only 15 SCLC cases were diagnosed (5.8\%), a percentage very similar to our results, where SCLC comprises $6 \%$ of all diagnosed cases [15]. Other studies, such as that published by Kreuzer et al. [16, 17] in Germany, have 
TABLE 1 Sample description

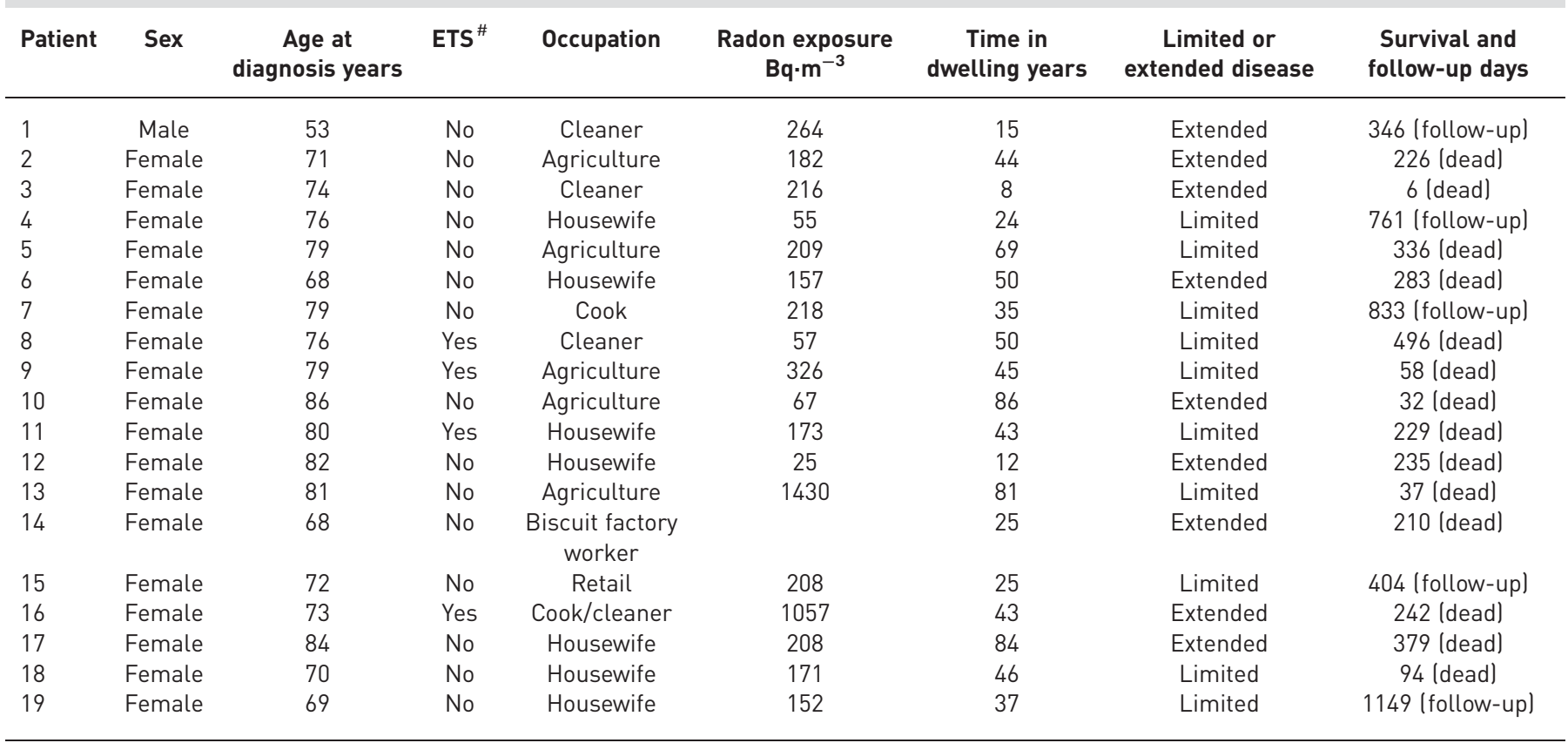

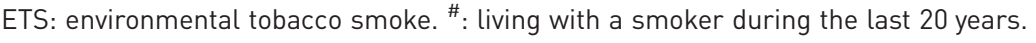

observed a higher percentage of SCLC, reaching 13.7\% in women [17] and 15.5\% in men [16]. SCLC has been related to exposure to tobacco smoke and this is the reason why it is linked to smokers. Passive smoking, which is a recognised human carcinogen [18], might contribute as a higher risk of SCLC in never-smokers. In our series only four (21\%) out of 19 cases had lived with a smoker in the last 20 years.

Previous studies have analysed the possible relationship between residential radon exposure, which is the main risk factor for lung cancer in never-smokers [19], and histological type, although most of these investigations included smokers and ex-smokers. The WHO has established a radon action level of $100 \mathrm{~Bq} \cdot \mathrm{m}^{-3}[19]$, while median residential radon in our case-series was $195 \mathrm{~Bq} \cdot \mathrm{m}^{-3}$. The European pooling study observed a linear increase in lung cancer risk for residential radon exposure [20] and a significant risk for lung cancer departing from $200 \mathrm{~Bq} \cdot \mathrm{m}^{-3}$. The present results are in line with available data on the relationship between radon and lung cancer. Regarding if residential radon might affect the presence or not of extended disease at diagnosis, we did not find any difference in radon concentrations between patients with limited disease compared with those with extended disease.

Lung cancer signs and symptoms are unspecific and, frequently, when first symptoms appear, the disease is at an advanced stage. Most patients in our study presented symptoms related to local affectation (cough, haemoptysis, dyspnoea) probably due to the central location characteristic of this type of tumour that can affect central large bronchi and cause these symptoms, with cough being the most frequent. All patients with systemic clinical manifestations at diagnosis suffered from extended disease.

$\begin{array}{lc}\text { TABLE } 2 \text { Symptoms at diagnosis } & \\ \text { Constitutional syndrome } & 5(26.3) \\ \text { Cough } & 11(57.9) \\ \text { Haemoptysis } & 5(26.3) \\ \text { Dyspnoea } & 6(31.6) \\ \text { Thoracic pain } & 7(36.8) \\ \text { Symptoms of metastasis } & 1(5.3) \\ \text { Asymptomatic } & 1(5.3)\end{array}$

Data are presented as $\mathrm{n}(\%) .{ }^{*}$ : as some patients can present more than one symptom, the total of all symptoms is greater than the number of patients included $(n=19)$. 
TABLE 3 Performance status (Eastern Cooperative Oncology Group Study) [10] by stage at diagnosis

\begin{tabular}{lccccc} 
& \multicolumn{5}{c}{ Performance status } \\
\cline { 2 - 6 } & $\mathbf{0}$ & $\mathbf{1}$ & $\mathbf{2}$ & $\mathbf{3}$ & $\mathbf{4}$ \\
\hline Limited disease & $3(30)$ & $4(40)$ & $1(10)$ & $0(0)$ & $2(20)$ \\
Extended disease & $1(11.1)$ & $4(44.4)$ & $0(0)$ & $3(33.3)$ & $1(11.1)$ \\
Total patients & $4(21.1)$ & $8(42.1)$ & $1(5.3)$ & $3(15.8)$ & $3(15.8)$ \\
\hline
\end{tabular}

Data are presented as $\mathrm{n}(\%)$.

Our SCLC patients were older than never-smoking lung cancer patients included in the LCRINS study [9] that were diagnosed with adenocarcinoma or large cell carcinoma. Median age at SCLC diagnosis was 75 years (IQR 70-80 years), whereas in the LCRINS study median age at diagnosis was 69 years (IQR 6077 years) for adenocarcinoma $(n=253)$ and 65 years (IQR 50-73 years) for large cell carcinoma $(n=8)$. Median age for squamous cell carcinoma $(n=30)$ was similar to SCLC: 76 years (IQR 65-82 years). Our SCLC patients are also older than those included in a similar case-series (with 19 never-smoking SCLC cases) published by VARGHeSE et al. [21], where median age at diagnosis was 65 years (range 35-94 years). We are not aware of any study that has analysed the relationship between lung cancer histological type and age at diagnosis in never-smokers, even when age at lung cancer diagnosis in never-smokers is an issue that has been addressed by several authors in different geographical settings. It seems that Asiatic never-smokers with lung cancer are diagnosed younger than ever-smokers. Nevertheless, in Europe and in the USA there is no difference in age at diagnosis between never- and ever-smokers, and never-smokers are even slightly older than ever-smokers in some series [22].

The differences observed in SCLC incidence for males and females deserve a comment. In the LCRINS study [15] the proportion of women is $\sim 80 \%$ and this is a gender distribution similar to the observed in other studies which included only never-smokers [23]. These differences favouring women are logical because most men are ever-smokers, while in Europe (and especially in Spain), older cohorts or women are mainly never-smokers. For all histological types there exist a higher number of women than men, but the male/female ratio varies depending on the tumour type. Our male/female ratio was $1 / 4.5$ for adenocarcinoma, 1/2 for squamous cell carcinoma and 1/1.6 for large cell carcinoma. The most surprising result was observed for SCLC, with a ratio 1/18 that is clearly higher than that observed in similar case-series recently published, such as that by VARGHESE et al. [21] with a $1 / 1$ ratio. In a previous study including 15 SCLC cases [14], the male/female ratio was $2.75 / 1$. We can only hypothesise about the causes of the high male/female difference observed in our study, and perhaps radon exposure might contribute to these differences because women spend more time at home than men (higher prevalence of housewives) and therefore higher radon exposure or since that women have a higher life expectancy than men, the chance of SCLC in never-smokers is higher for them, especially with a median age at diagnosis of 75 years.

SCLC survival is very low. In limited stages the median survival is $\sim 15-20$ months, with a 2 -year survival of $20-40 \%$ [24], whereas in metastatic disease survival is $<10$ months [25]. In our series of never-smokers, median survival was lower, at 11.2 months in patients with limited diseases and 7.8 months in patients with extended disease. Stage at diagnosis and performance status are well-known prognostic factors in all histologic types of lung cancer [26], and also specifically in SCLC [27, 28]. In a recently published study [29] that analysed the clinical usefulness of the available prognostic tools in lung cancer (including NSCLC and SCLC), performance status was the only predictive factor that was present in all tools, while stage at diagnosis was used in five of the tools. The present results confirm the relationship between stage at diagnosis, performance status and survival in never-smoking patients with SCLC.

In the present study, EGFR (epidermal growth factor receptor), KRAS or ALK (anaplastic lymphoma kinase) mutations have not been determined since they are very infrequent in SCLC [30]. These mutations usually appear in other histological types, such as adenocarcinoma, and various scientific societies have published guidelines on how and when these should be determined in advanced NSCLC. There is no evidence to support the detection of these mutations in SCLC [31].

This research has some advantages. The main advantage is the multicenter nature of this study, which has allowed inclusion of a relatively high number of cases, given the low frequency of SCLC in never-smokers. We have collected information on the most important and known risk factors for lung cancer in never-smokers, including residential radon exposure, ETS exposure and occupation. To the best of our knowledge, this is the first study that has assessed residential radon concentration individually in a sample 
of never-smokers with SCLC. This study has also been performed in a radon-prone area, while the remaining studies have not been performed in such radon risk areas.

The present study has also many limitations. Due to the limited sample size we only have included one male and perhaps the results would differ if we had more males in our sample. In fact, we cannot disregard a gender preference for SCLC in never-smokers for females. Hormonal factors might play some role in its induction. We have not been able to obtain information from other risk factors such as family history of cancer.

To conclude, SCLC in never-smokers is very infrequent and is a highly aggressive disease, similar to SCLC occurring in ever-smokers. Survival is very poor, even in cases diagnosed with a limited disease. Age at diagnosis in SCLC is higher than that observed for never-smokers with adenocarcinoma. Residential radon exposure in these cases is higher than the action levels recommended by the WHO and higher than that observed for the general population in the same area. More studies are necessary to better characterise this disease in never-smokers in order to obtain a deeper understanding of its risk factors combined with the biological characteristics of this tumour.

\section{References}

1 Jemal A, Bray F, Center MM, et al. Global cancer statistics. CA Cancer J Clin 2011; 61: 69-90.

2 Devesa SS, Bray F, Vizcaino AP, et al. International lung cancer trends by histologic type: male/female differences diminishing and adenocarcinoma rates rising. Int J Cancer 2005; 117: 294-299.

3 Hoffman D, Hoffman I. The changing cigarette 1950-1995. J Toxicol Environ Health 1997; 50: 307-364.

4 Travis W, Brambilla E, Muller-Hermelink H, et al. editors. World Health Organization classification of tumours. Pathology and genetics of tumours of the lung, pleura, thymus and heart. Lyon, IARC Press, 2004.

5 Früh M, De Ruysscher D, Popat S, et al. Small-cell lung cancer (SCLC): ESMO Clinical Practice Guidelines for diagnosis, treatment and follow-up. Ann Oncol 2013; 24: Suppl. 6, vi99-v105.

6 Ou S-HI, Ziogas A, Zell JA. Prognostic factors for survival in extensive stage small cell lung cancer (ED-SCLC): the importance of smoking history, socioeconomic and marital statuses, and ethnicity. J Thorac Oncol 2009; 4: $37-43$.

7 Kim CH, Lee Y-CA, Hung RJ, et al. Exposure to secondhand tobacco smoke and lung cancer by histological type: a pooled analysis of the International Lung Cancer Consortium (ILCCO). Int J Cancer 2014; 135: 1918-1930.

8 Barros-Dios JM, Ruano-Ravina A, Pérez-Ríos M, et al. Residential radon exposure, histologic types, and lung cancer risk. A case-control study in Galicia, Spain. Cancer Epidemiol Biomark Prev 2012; 21: 951-958.

9 Torres-Durán M, Ruano-Ravina A, Parente-Lamelas I, et al. Residential radon and lung cancer characteristics in never smokers. Int J Radiat Biol 2015; 91: 605-610.

10 Oken MM, Creech RH, Tormey DC, et al. Toxicity and response criteria of the Eastern Cooperative Oncology Group. Am J Clin Oncol 1982; 5: 649-655.

11 Vargas A, Ortega X. Influence of environmental changes on integrating radon detectors: results of an intercomparison exercise. Radiat Prot Dosimetry 2007; 123: 529-536.

12 Gutierrez-Villanueva JL, Sainz-Fernández C, Fuente-Merino I, et al. Intercomparison exercise on external gamma dose rate under field conditions at the laboratory of natural radiation (Saelices el Chico, Spain). Radiat Prot Dosimetry 2013; 155: 459-466.

13 Khakwani A, Rich AL, Tata LJ, et al. Small-cell lung cancer in England: trends in survival and chemotherapy using the National Lung Cancer Audit. PLoS One 2014; 9: e89426.

14 Lagarde F, Axelsson G, Damber L, et al. Residential radon and lung cancer among never-smokers in Sweden. Epidemiology 2001; 12: 396-404.

15 Torres-Durán M, Ruano-Ravina A, Parente-Lamelas I, et al. Lung cancer in never smokers. A case-control study in a radon prone area (Galicia, Spain). Eur Respir J 2014; 44: 994-1001.

16 Kreuzer M, Gerken M, Kreienbrock L, et al. Lung cancer in lifetime nonsmoking men - results of a case-control study in Germany. Br J Cancer 2001; 84: 134-140.

17 Kreuzer M, Heinrich J, Kreienbrock L, et al. Risk factors for lung cancer among nonsmoking women. Int J Cancer 2002; 100: 706-713.

18 Trédaniel J, Boffetta P, Saracci R, et al. Exposure to environmental tobacco smoke and risk of lung cancer: the epidemiological evidence. Eur Respir J 1994; 7: 1877-1888.

19 World Health Organization. Handbook on indoor radon: a public health perspective. Geneva, WHO, 2009.

20 Darby S, Hill D, Auvinen A, et al. Radon in homes and risk of lung cancer: collaborative analysis of individual data from 13 European case-control studies. BMJ 2005; 16: 137-145.

21 Varghese AM, Zakowski MF, Yu HA, et al. Small-cell lung cancers in patients who never smoked cigarettes. J Thorac Oncol 2014; 9: 892-896.

22 Sun S, Schiller JH, Gazdar AF. Lung cancer in never smokers - a different disease. Nat Rev Cancer 2007; 7: 778-790.

23 Lo Y-L, Hsiao CF, Chang GC, et al. Risk factors for primary lung cancer among never smokers by gender in a matched case-control study. Cancer Causes Control 2013; 24: 567-576.

24 van Meerbeeck JP, Fennell DA, De Ruysscher DKM. Small-cell lung cancer. Lancet 2011; 378: 1741-1755.

25 Foster NR, Qi Y, Shi Q, et al. Tumor response and progression-free survival as potential surrogate endpoints for overall survival in extensive stage small-cell lung cancer: findings on the basis of North Central Cancer Treatment Group trials. Cancer 2011; 117: 1262-1271.

26 Buccheri G, Ferrigno D. Prognostic factors in lung cancer: tables and comments. Eur Respir J 1994; 7: 1350-1364.

27 Vincent MD, Ashley SE, Smith IE. Prognostic factors in small cell lung cancer: a simple prognostic index is better than conventional staging. Eur J Cancer Clin Oncol 1987; 23: 1589-1599. 
28 Maestu I, Pastor M, Gómez-Codina J, et al. Pretreatment prognostic factors for survival in small-cell lung cancer: a new prognostic index and validation of three known prognostic indices on 341 patients. Ann Oncol 1997; 8: 547-553.

29 Mahar AL, Compton C, McShane LM, et al. Refining prognosis in lung cancer: a report on the quality and relevance of clinical prognostic tools. J Thorac Oncol 2015; 10: 1576-1589.

30 Herbst RS, Heymach JV, Lippman SM. Lung cancer. N Engl J Med 2008; 359: 1367-1380.

31 Garrido P, de Castro J, Concha Á, et al. Guidelines for biomarker testing in advanced non-small-cell lung cancer. A National Consensus of the Spanish Society of Medical Oncology (SEOM) and the Spanish Society of Pathology (SEAP). Clin Transl Oncol 2012; 14: 338-349. 